\title{
Dos nuevas especies de cangrejos de agua dulce (Brachyura: Pseudothelphusidae) de la Serranía de Los Paraguas, Cordillera Occidental de Colombia
}

\author{
Gabriel E. Ramos-Tafur \\ 3540 32nd Ave. North, Apt. 216, Saint Petersburg, FL. 33713, EEUU; gabrieleramos1@hotmail.com
}

Recibido 09-XII-2002. Corregido 12-I-2004. Aceptado 29-XI-2005.

\begin{abstract}
Two new species of fresh water crab (Brachyura: Pseudothelphusidae) from Serranía de Los Paraguas, Cordillera Occidental, Colombia. Two new species of freshwater crabs of the genus Hypolobocera from Serranía de Los Paraguas, Cordillera Occidental, Colombia, are described. Hypolobocera solimani n. sp. is closely related to H. alata Campos 1989, but can be distinguished from it by smooth upper margin of third maxilliped merus, the size and shape of apex of first gonopod, and the internal lobe or papilla of spermatic channel, which is concave, raised, with ends with minute papillae or rounded tubercles. Hypolobocera triangula n. sp. is very similar to H. rotundilobata Rodríguez 1994, but differs from it by the shape and size of the lateral lobe, an oblique basal ridge of tubercles and by the apex of first gonopod. With the two new species, the total number of valid members of the genus Hypolobocera known from western Colombia or biogeographic Chocó is 20. Rev. Biol. Trop. 54 (3): 1047-1056. Epub 2006 Sept. 29.
\end{abstract}

Key words: Hypolobocera solimani, Hypolobocera triangula, freshwater crabs, Colombia, Pseudothelphusidae, new species.

Las características morfológicas externas de los cangrejos de agua dulce de la familia Pseudothelphusidae, tales como la forma del caparazón y la presencia de dientes, espinas, tubérculos o papilas, presentan alta variabilidad, aún intraespecífica, en diferentes géneros, por lo que son utilizadas más como caracteres secundarios en la descripción de algunas especies (Magalhães y Türkay 1996). Türkay (1975) informó acerca de la morfología del primer par de pleópodos de los machos, anotando que estas estructuras son las menos afectadas por los cambios medioambientales y su funcionalidad esta ligada exclusivamente al momento de la transferencia del espermatóforo a la hembra, cuando estos apéndices interactúan con la armadura reproductiva femenina conformando un sistema complejo. Los cambios básicos en la morfología del gonópodo solamente pueden ocurrir si el ducto de la hembra evoluciona en la misma dirección. La coevolución de dos estructuras morfológicamente diferentes (internas) es definitivamente más lenta que cualquier otro de los caracteres externos afectados por el medio ambiente. Por lo tanto, la clasificación sistemática de este grupo se basa principalmente en la morfología externa del gonópodo.

La revisión de un lote de cangrejos de agua dulce, recolectados en una misma localidad del occidente de Colombia, permitió descubrir que, aunque exteriormente los cangrejos lucen similares, el estudio de sus gonópodos demostró la presencia de dos nuevas especies de Hypolobocera cohabitando en simpatría, las cuales son objeto de descripción en el presente estudio. 


\section{MATERIALES Y MÉTODOS}

El material proviene de una recolección de 20 especimenes de cangrejos de agua dulce, efectuada durante una salida de campo del curso de mastozoología de la Universidad del Valle. El muestreo se efectuó en una zona selvática y altamente húmeda, denominada localmente como Paso de Galápagos, ubicada en la parte alta de la Serranía de Los Paraguas, Cordillera Occidental, entre los límites de los departamentos de Chocó y Valle del Cauca, Colombia. El material se fijó y etiquetó en el campo y posteriormente se depositó en la Sección de Biología Marina de la misma universidad.

Se inició un estudio minucioso, separando, comparando e identificando los cangrejos, con el material tipo depositado en la Colección de la Universidad del Valle y las descripciones e ilustraciones de las especies que han sido descritas o informadas de lugares adyacentes por: Rathbun (1905), Pretzmann (1965, 1968), Rodríguez (1980, 1982, 1992, 1994), Prahl (1983, 1985, 1987a, 1987b, 1988a, 1988b), Prahl y Giraldo (1985), Campos (1989, 2003), Campos y Rodríguez (1995) y Rodríguez y Sternberg (1998). Este análisis arrojó el descubrimiento de dos nuevas especies.

A ambas especies se les disectó y dibujó el primer gonópodo izquierdo. La terminología utilizada para la descripción del gonópodo es la sugerida por Smalley (1964) y Rodríguez (1982). Las abreviaturas utilizadas en el texto son: $\mathrm{AC}=$ ancho del caparazón; LC= largo del caparazón; cuando más de un espécimen fue medido, se presenta el intervalo entre el máximo y el mínimo, en milímetros; CRBMUV= Colección de Referencia de Biología Marina de la Universidad del Valle, Cali, Colombia; USNM= National Museum of Natural History, Smithsonian Institution, Washington, D.C., EEUU.

Hypolobocera solimani sp. nov.

(Fig. 1-2)

Material examinado: $1 \sigma^{7}$ holotipo, AC $20.4 \mathrm{~mm}$, LC $13.0 \mathrm{~mm}$, gonópodo $5.1 \mathrm{~mm}$; 1 q alotipo, AC $21.0 \mathrm{~mm}$, LC 13.9 mm; Paso de
Galápagos, Serranía de Los Paraguas (447’30”' $\left.\mathrm{N}, 76^{\circ} 09^{\prime} 02^{\prime \prime} \mathrm{W}\right), 2500 \mathrm{msnm}$, aproximadamente $11 \mathrm{~km}$ al NE del Municipio de El Cairo, Departamento del Valle del Cauca, Colombia, 15 mayo 1985, recolectores M. Alberico y L. Neira, (CRBMUV 85147).

English diagnosis: Carapace with upper border of front smooth, median groove short and inconspicuos. Anterolateral margin with small and numerous papillae. Cervical groove shallow, slightly arched. Large chela with dactylus shorter than fixed finger. First gonopod with lateral lobe situated distally, close to apex, subtriangular in shape. Contour of apex irregularly shaped; lateral border with conspicuous tooth and papillar protuberance; caudal margin with five small triangular teeth; internal lobe or central papilla concave, raised, with end rounded and bordered by small knobs or minute papillae.

Diagnosis: Caparazón con margen frontal superior liso, surco medio corto y superficial. Margen anterolateral con pequeñas y numerosas papilas. Surco cervical somero, ligeramente arqueado. Quela mayor con el dáctilo más corto que el dedo fijo. Primer gonópodo con lóbulo lateral ubicado distalmente, cerca del ápice, de forma subtriangular. Contorno del ápice de forma irregular, borde lateral con un diente conspicuo y protuberancia papilar; margen caudal con cinco dientes triangulares pequeños; lóbulo interno o papila central cóncavo, proyectado hacia arriba, con extremo redondeado, bordeado por pequeñas protuberancias o diminutas papilas.

Descripción: Regiones dorsales del caparazón pobremente definidas, lóbulos postfrontales pequeños, ligeramente demarcados por dos pequeñas elevaciones; surco medio corto y mal definido; surco cervical somero, ligeramente curvo, el cual no alcanza el borde anterolateral (Fig. 1A). Margen orbital liso, sinuoso; diente orbital subtriangular, seguido de pequeñas papilas irregulares; primer diente anterolateral poco desarrollado; margen anterolateral con numerosas papilas pequeñas. Abertura del canal branquial eferente cóncava, 

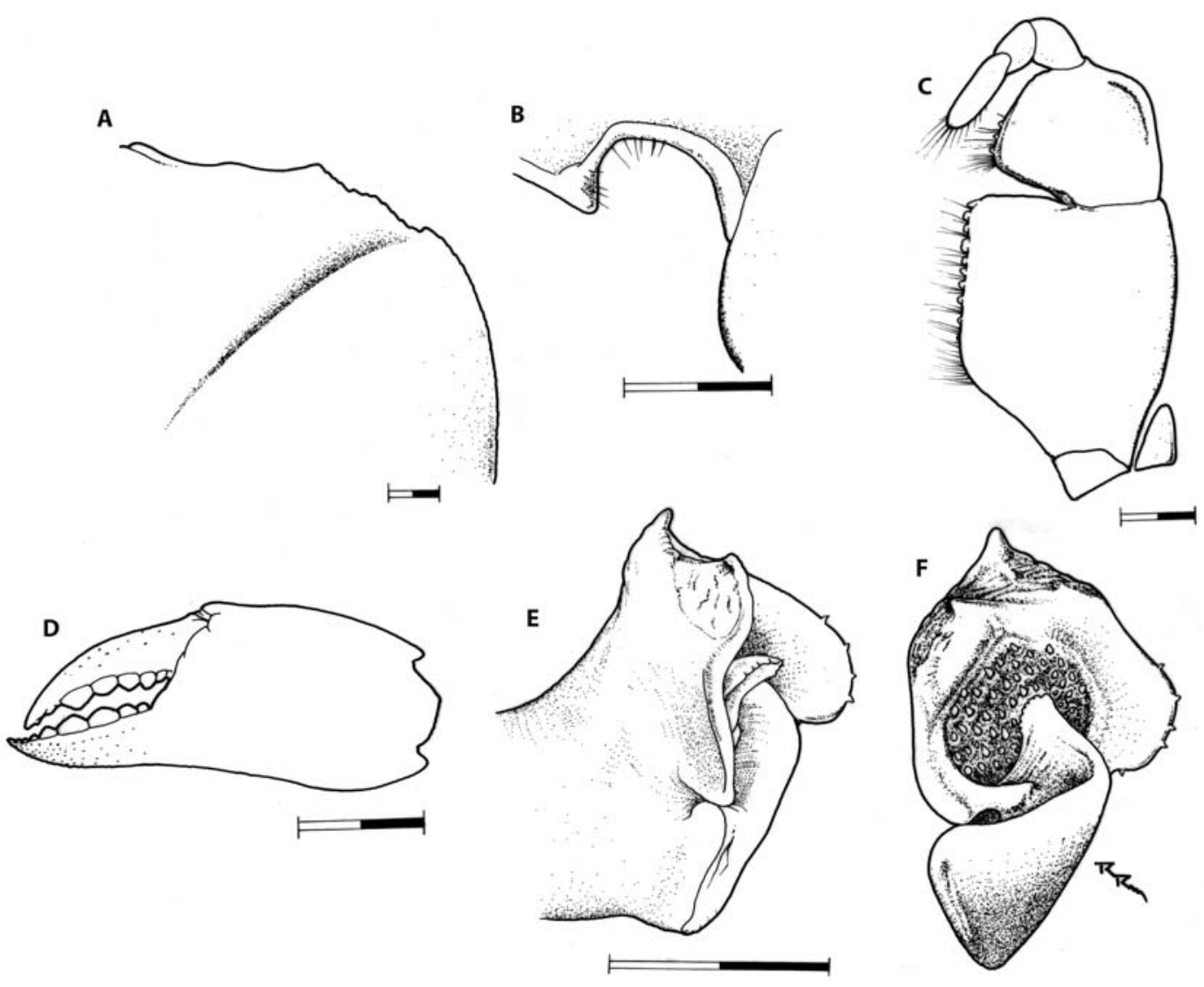

Fig. 1. Hypolobocera solimani sp. nov., ơ holotipo, AC 20.4 mm, LC 13.0 mm, gonópodo 5.1 mm, Paso de Galápagos, Serranía de Los Paraguas, límite entre los departamentos del Chocó y Valle del Cauca, Colombia (CRBMUV 85147). A. Detalle del borde anterolateral del caparazón, vista dorsal. B. Abertura del canal branquial eferente, vista frontal. C. Tercer maxilípedo, vista frontal. D. Quela mayor, vista externa. E. Detalle de la porción apical del gonópodo izquierdo, vista mesial. F. Mismo, vista apical. Escalas A-C, E-F $=1 \mathrm{~mm}, \mathrm{D}=5 \mathrm{~mm}$.

Fig. 1. Hypolobocera solimani new species, ơ holotype, CB 20.4 mm, CL 13.0 mm, gonopod 5.1 mm, Paso de Galápagos, Serranía de Los Paraguas, between Chocó and Valle del Cauca, Colombia (CRBMUV 85147). A. Anterolateral border of carapace, dorsal view. B. Aperture of efferent branchial channel, frontal view. C. Third maxilliped, frontal view. D. Major chela, external view. E. Left gonopod, detail of apex, mesial view. F. Same, superior view. Scales A-C, E-F = $1 \mathrm{~mm}, \mathrm{D}=5 \mathrm{~mm}$.

en forma de $\mathrm{C}$ amplia (Fig. 1B), margen proximal ligeramente proyectado, con pocas setas. Margen interno del mero del tercer maxilípedo con tres dientes pequeños y mechones de setas intercalados, margen externo liso; isquio con 10 dientes aplanados y algunas setas; exognato cerca de 0.25 veces en la longitud del isquio del endognato (Fig. 1C).

Primer par de pereópodos presentando heteroquelia. Quela mayor con la palma inflada y tubérculos pequeños o puntuaciones sobre la superficie de los dedos; dientes de la superficie cortante de ambos dedos subtriangulares. Sin tubérculos en la cara externa del dedo fijo y el dedo móvil. Dáctilo mas corto que el dedo fijo, tanto en el macho como en la hembra (Fig. 1D).

Contorno del ápice del gonópodo de forma irregular, inclinado en un ángulo aproximado de $45^{\circ}$ sobre el margen cefálico. Proceso caudal proyectado por encima de la superficie del ápice, con cinco dientes triangulares pequeños 

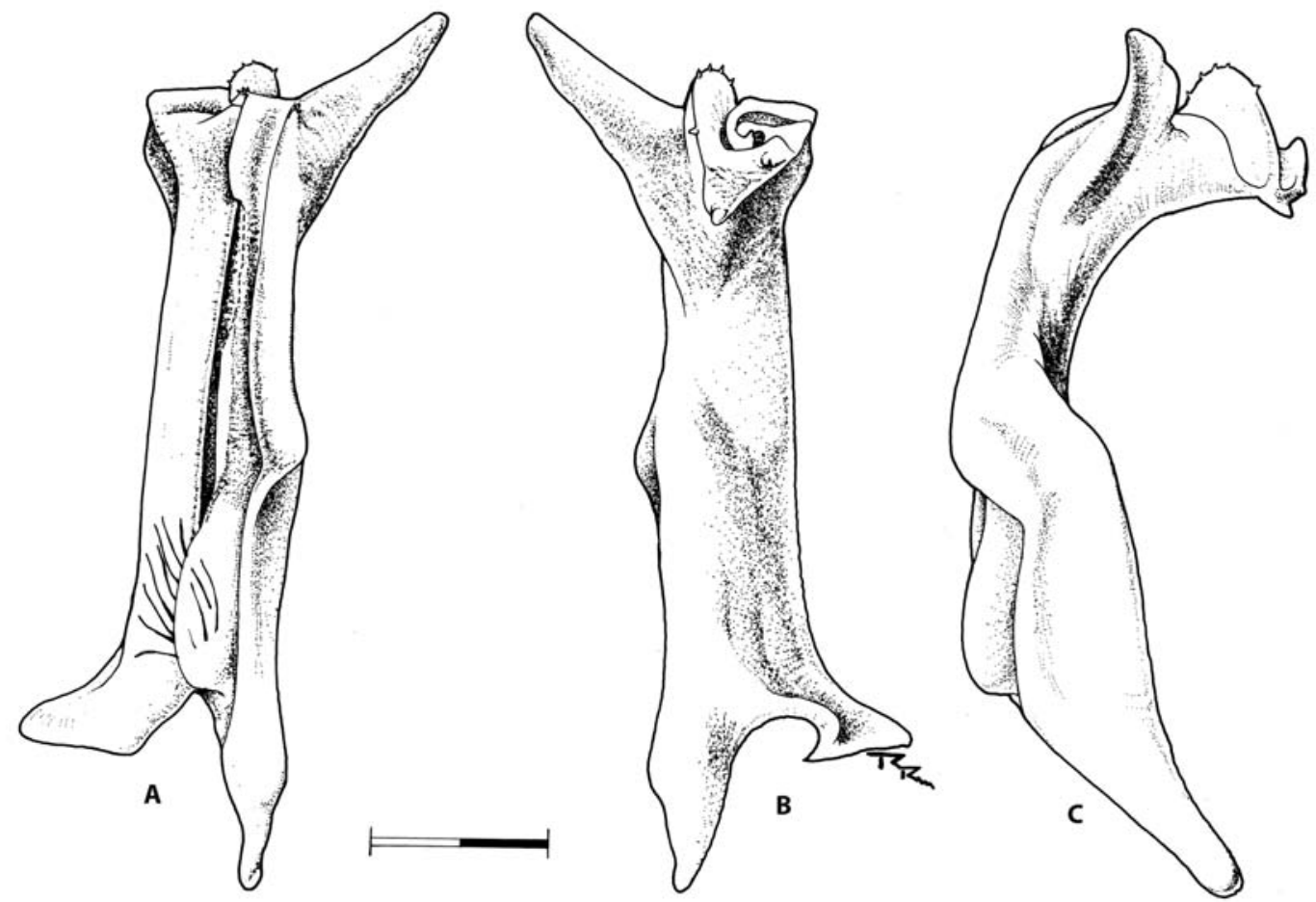

Fig. 2. Hypolobocera solimani sp. nov., holotipo, gonópodo izquierdo, $5.1 \mathrm{~mm}$ (CRBMUV 85147). A. Vista caudal. B. Vista cefálica. C. Vista lateral. Escala $=1 \mathrm{~mm}$.

Fig. 2. Hypolobocera solimani new species, holotype, left gonopod, $5.1 \mathrm{~mm}$ (CRBMUV 85147). A. Caudal view. B. Cephalic view. C. Lateral view. Scale $=1 \mathrm{~mm}$.

sobre su borde (Fig. 1E-F); margen cefálico casi recto, con borde liso; lóbulo mesial subtriangular con extremos redondeados; borde lateral irregular, terminando en forma de un gran diente de punta roma y curvada hacia arriba, protuberancia papilar en forma de diente laminar obtuso, dirigido hacia el borde cefálico; lóbulo interno o papila del canal espermático cóncavo, redondeado, proyectado hacia arriba, en forma de cuchara, cubriendo parte del campo de espinas, con el extremo terminando en pequeñas protuberancias romas o diminutas papilas. Gonópodo casi recto en vista caudal; lóbulo lateral desplazado hacia el extremo apical, protuberante, de forma subtriangular y punta roma (Fig. 2A-B); margen caudal con escasas setas a nivel proximal; cresta caudal conspicua, visible en vista lateral (Fig. 2C), globosa a nivel central, la cual continua ligeramente inflada y se vuelve casi recta, hasta cerca de la base del lóbulo lateral.

Etimología: Se nombra en honor del amigo Arnulfo Soliman Cuero (1972-1999), Capitán de lancha, compañero de agotadoras jornadas de trabajo de campo e infatigable trabajador del Instituto Nacional de Pesca y Acuícultura (INPA), regional de Buenaventura.

Coloración: Dorso del caparazón café claro o amarillo, con máculas café oscuro sobre las regiones más protuberantes. Pereópodos y quelípedos café claro, con máculas café oscuro sobre las articulaciones. Puntas de los dáctilos negras.

Hábitat: Los cangrejos fueron recolectados en un ríachuelo (al parecer sin nombre específico), en madrigueras y en pequeñas 
charcos, ocultos debajo de vegetación húmeda. Sustrato fangoso, con predominio de abundante materia orgánica.

Observaciones: Rodríguez (1982) propuso la subdivisión del género Hypolobocera en seis grupos, basado principalmente en la morfología del gonópodo, longitud del exognato del tercer maxilípedo y en sus patrones biogeográficos. Campos (2003) en su reciente revisión del género, sugiere la creación de dos grupos adicionales para incluir en el grupo siete a $H$. alata Campos 1989 y grupo ocho para $H$. kamsarum Campos \& Rodríguez 1995, especies que anteriormente eran consideradas como aberrantes o sin grupo.

Acorde con esta dos ultimas subdivisiones artificiales del género, la morfología externa del gonópodo de Hypolobocera solimani sp. nov. la ubica dentro del grupo siete, presentando un gonópodo morfológicamente muy similar al exhibido por su congénere $H$. alata Campos. Las principales características para poder diferenciar estas dos especies son: $H$. alata presenta una muesca en el margen externo del mero del tercer maxilípedo, mientras que $H$. solimani tiene el margen externo del mero del tercer maxilípedo liso. El gonópodo de $H$. alata exhibe el margen caudal del ápice, armado con cuatro dientes pequeños, es redondeado y se hace casi recto hacia el diente lateral; el margen cefálico es suavemente redondeado; el margen mesial tiene sus bordes irregulares o sinuosos y lóbulo interno o papila del canal espermático termina en una papila bífida. Por su parte el gonópodo de $H$. solimani presenta el margen caudal del ápice ornamentado con cinco dientes triangulares pequeños, es notoriamente abultado o globoso y se continua redondeado hacia el diente lateral; el margen cefálico es casi recto; el margen mesial es liso; el lóbulo interno o papila del canal espermático es cóncavo, elevado, en forma de cuchara (en vista lateral) y termina rebordeado por pequeñas protuberancias o diminutas papilas.

Campos (1989) informó a $H$. alata de Quebrada La Batea, Municipio de Villavieja, Departamento del Huila a $400 \mathrm{msnm}$, en el
Valle del Río Magdalena, entre las Cordilleras Central y Oriental de Colombia (localidad tipo), mas tarde Campos (2003), anota que esta localidad puede ser errónea y rectifica que la distribución de esta especie es entre Puerto Rico, Risaralda y Tadó, Chocó, a altitudes entre 490 y $700 \mathrm{msnm}$, H. solimani solo se ha recolectado en la localidad tipo a 2500 msnm.

\section{Hypolobocera triangula sp. nov.}

(Fig. 3-4)

Hypolobocera rotundilobata: Campos 2003: 794 (parte, solo el material depositado en la CRBMUV), no Hypolobocera rotundilobata Rodríguez 1994.

Material examinado: $1 \sigma^{\gamma}$ holotipo, 1 q alotipo, Paso de Galápagos, Serranía de Los Paraguas (4⒋'30’'N, 7609'02”' W), 2500 msnm, aproximadamente $11 \mathrm{~km}$ al NE del Municipio de El Cairo, Departamento del Valle del Cauca, Colombia, 15 mayo 1985, recolectores M. Alberico y L. Neira. Paratipos: $5 \sigma^{7}$, 7 (CRBMUV 85148); 2 ○', 2 ○ (USNM 270046).

Medidas: Machos, AC 11.0-24.5 mm, LC 7.7-19.1 mm, gonópodo del holotipo $5.2 \mathrm{~mm}$; hembras, AC 17.3-23.2 mm, LC 10.5-14.1 mm.

English diagnosis: Dorsal surface of carapace poorly delineated. Median groove shallow and narrow. Anterolateral margin with 15-17 small teeth. Cervical groove shallow, almost straight. Fixed finger of major cheliped without tubercles on external base. First gonopod with lateral lobe inflated, smooth, slightly rounded; basal ridge oblique with nine small knobs. Apex subtriangular in shape; papillar protuberance shaped as an acute tooth, tip indented by three small tubercles; row of acute papillae along cephalic border.

Diagnosis: Caparazón con áreas dorsales pobremente definidas. Surco medio somero y estrecho. Margen anterolateral con 15-17 dientes pequeños. Surco cervical superficial, casi 


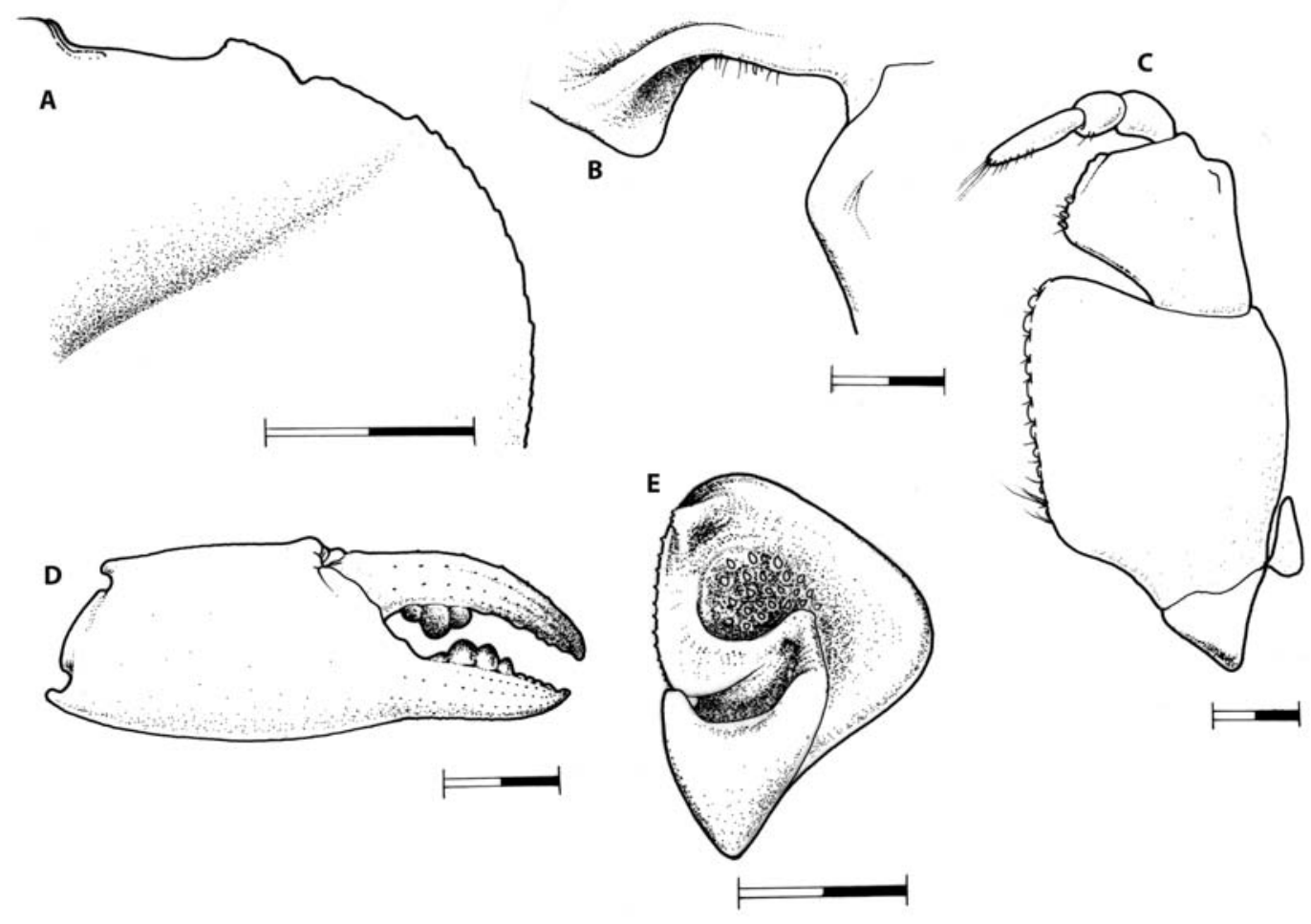

Fig. 3. Hypolobocera triangula sp. nov., $\sigma^{\Upsilon 7}$ holotipo, AC= 24.5 mm, LC= 19.1 mm, gonópodo 5.2 mm, Paso de Galápagos, Serranía de Los Paraguas, límite entre los departamentos del Chocó y Valle del Cauca, Colombia (CRBMUV 85148). A. Detalle del borde anterolateral del caparazón, vista dorsal. B. Abertura del canal branquial eferente, vista frontal. C. Tercer maxilípedo, vista frontal. D. Quela mayor, vista externa. E. Gonópodo izquierdo, vista apical. Escalas A, D = 5 mm, B-C, $\mathrm{E}=1 \mathrm{~mm}$.

Fig. 3. Hypolobocera triangula new species, $\mathrm{O}^{7}$ holotype, $\mathrm{AC}=24.5 \mathrm{~mm}, \mathrm{LC}=19.1 \mathrm{~mm}$, gonópodo $5.2 \mathrm{~mm}$, Paso de Galápagos, Serranía de Los Paraguas, between Chocó and Valle del Cauca, Colombia (CRBMUV 85148). A. Anterolateral border of carapace, dorsal view. B. Aperture of branchial efferent channel, frontal view. C. Third maxilliped, frontal view. D. Major chela, external view. E. Left gonopod, superior view. Scales A, D = $5 \mathrm{~mm}, \mathrm{~B}-\mathrm{C}, \mathrm{E}=1 \mathrm{~mm}$.

recto. Dedo fijo del quelípedo mayor sin tubérculos en la base externa. Gonópodo con lóbulo lateral inflado, liso, ligeramente redondeado; cresta basal oblicua con nueve pequeñas protuberancias. Ápice de forma subtriangular; protuberancia papilar en forma de diente agudo, con tres tubérculos pequeños en su cima; borde cefálico con una hilera de papilas agudas.

Descripción: Caparazón con regiones dorsales no protuberantes, superficie lisa; lóbulos postfrontales pequeños, ligeramente inflados por encima de la superficie del caparazón; surco medio somero y estrecho; surco cervical casi recto, superficial, el cual no alcanza el borde anterolateral (Fig. 3A); margen orbital casi liso con tubérculos diminutos; diente orbital cuadrangular; primer diente anterolateral poco desarrollado; margen anterolateral con 15-17 dientes pequeños, los cuales disminuyen de tamaño posteriormente. Abertura del canal branquial eferente en forma de $U$ ancha, con márgenes subparalelos y setas escasas en el margen superior (Fig. 3B). Margen interno del mero del tercer maxilípedo con tres dientes y setas escasas; extremo distal irregular con dos o tres protuberancias; margen externo con muesca en el extremo superior; isquio con 12 dientes aplanados, dos mechones de setas proximales y una o dos setas intercaladas entre cada diente 
(Fig. 3C); exognato aproximadamente 0.3 veces en la longitud del isquio del endognato.

Quelípedos desiguales; quela mayor abultada, con pequeños tubérculos o puntuaciones sobre la superficie de los dedos; en el holotipo los dientes se presentan de tamaño disparejo y están situados en la mitad próximal de la superficie cortante de los dedos (Fig. 3D), en el resto del material tipo los dientes son uniformes en tamaño y continuos a lo largo de toda la superficie. Sin tubérculos en la cara externa del dedo fijo o del dedo móvil.

Gonópodo con el contorno del ápice de forma subtriangular, lóbulo mesial en forma de corazón, con extremo angular; borde lateral amplio e hinchado hacia la región caudal, con protuberancia papilar proyectada hacia la región cefálica, la cual en su ápice presenta tres pequeños tubérculos romos; borde cefálico con una hilera de pequeñas y numerosas papilas espiniformes. Lóbulo interno o papila central proyectado sobre el campo de espinas, con extremo redondeado y desplazado ligeramente hacia el borde caudal (Fig. 3E). Lóbulo lateral liso, hinchado, de forma ligeramente subtriangular; con muesca superior en forma de $\mathrm{C}$ apartándolo del ápice y con profunda excavación en forma de $\mathrm{V}$ a nivel basal, cerca de la mitad del gonópodo (Fig. 4A, B); margen caudal con numerosas setas a nivel proximal; cresta caudal sinuosa, prominente, en forma de abultamiento globoso sobre la parte central, desapareciendo al aproximarse al ápice (Fig. 4C); proceso caudal abultado, ubicado por debajo del ápice, con pequeña excavación media; cresta basal oblicua, con nueve pequeñas protuberancias, la cual se extiende desde cerca de la mitad del lóbulo lateral hasta cerca de la mitad de la longitud del gonópodo (Fig. 4B), su parte inferior es visible parcialmente en vista caudal (Fig. 4A).
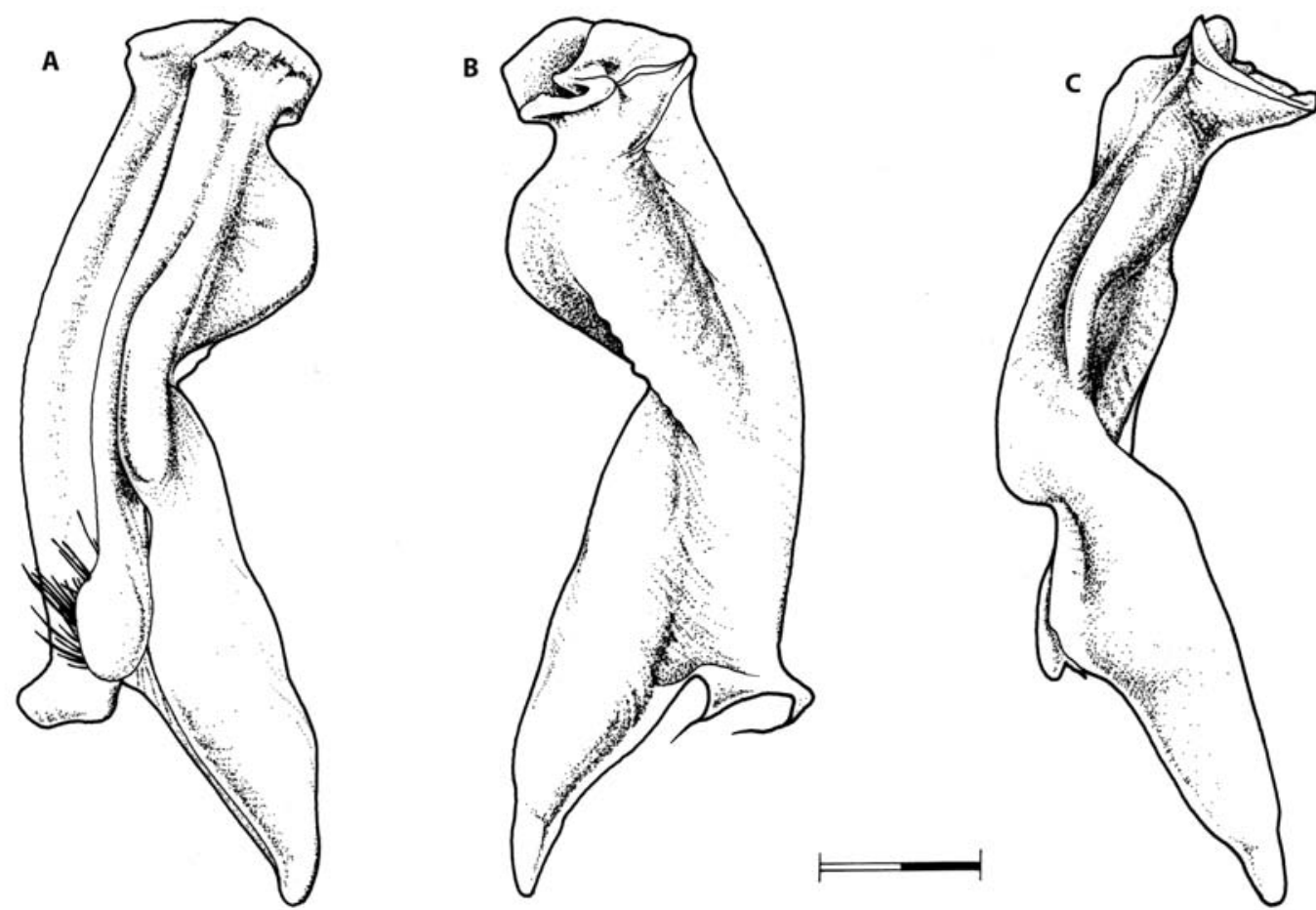

Fig. 4. Hypolobocera triangula sp. nov., holotipo, gonópodo izquierdo, 5.2 mm (CRBMUV 85148). A. Vista caudal. B. Vista cefálica. C. Vista lateral. Escala $=1 \mathrm{~mm}$.

Fig. 4. Hypolobocera triangula new species, holotype, left gonopod, $5.2 \mathrm{~mm}$ (CRBMUV 85148). A. Caudal view. B. Cephalic view. C. Lateral view. Scale $=1 \mathrm{~mm}$. 
Etimología: Del latín triangulus (triangular) y se refiere a la forma del contorno del ápice del gonópodo.

Coloración: Dorso del caparazón café oscuro, área ventral café claro; pereópodos y quelípedos sepia claro, intersección de los segmentos y puntas de los dáctilos negros.

Hábitat: Los cangrejos fueron recolectados en un riachuelo, en madrigueras y en pequeñas charcos, ocultos debajo de vegetación húmeda. Sustrato fangoso, con predominio de abundante materia orgánica.

Observaciones: Las fuertes excavaciones en la base y el margen superior del lóbulo lateral del gonópodo de $H$. triangula sp. nov., la hacen encajar dentro del grupo cuatro, de los propuestos por Rodríguez (1982), presentando similitudes morfológicas con $H$. rotundilobata Rodríguez 1994. Las principales características para diferenciar a estas dos especies son: el lóbulo lateral del gonópodo de $H$. rotundilobata es ligeramente redondeado sobre todo el margen; en cambio el lóbulo lateral de $H$. triangula es rústicamente subtriangular. $\mathrm{La}$ cresta caudal en $H$. rotundilobata es corta seguida por una elevación que se prolonga hasta cerca del ápice; en $H$. triangula la cresta caudal es alargada con una proyección en forma de joroba cerca del ápice. H. rotundilobata no presenta cresta basal debajo del lóbulo lateral o es inconspicua, mientras que $H$. triangula exhibe una cresta basal compuesta por nueve protuberancias pequeñas, organizadas de manera oblicua, las cuales se extienden desde la parte central del lóbulo lateral, hasta casi la parte central del gonópodo, donde desaparecen. Rodríguez (1994) en la figura 2B, vista lateral del gonópodo del holotipo de $H$. rotundilobata (USNM 240103), presenta una serie de arrugas transversales sobre el margen cefálico; Campos (2003) examinó el mismo espécimen (erróneamente lo señala depositado como USNM 240104), pero sus figuras 22B, D no presentan arruga alguna sobre el margen cefálico; en $H$. triangula el margen cefálico es liso, sin arrugas. El ápice del gonópodo de $H$. rotundilobata tiene el borde lateral recto, con papila redondeada y el borde cefálico es liso, sin espinas o papilas; en $H$. triangula el borde lateral es redondeado, la papila tiene en su cúspide tres tubérculos pequeños romos, el margen caudal es inflado a nivel central, y el borde cefálico tiene una serie de papilas espiniformes continuas. $H$. rotundilobata ha sido informado de Río San Juan, Playa de Oro (Rodríguez 1994), Rio Ingará, San José del Palmar, Departamento del Chocó, a altitudes entre 70 y 2000 msnm (Campos 2003); $H$. triangula solo se ha recolectado en la localidad tipo a 2500 msnm.

Campos (2003) informa dentro de su material examinado de $H$. rotundilobata a $2 \sigma^{7}$ y 6 , bajo el número CRBMUV 85147, pero este número en realidad corresponde al holotipo y alotipo de $H$. solimani. En el presente trabajo se informan $6 \sigma^{7}$ y 8 \& (material tipo de H. triangula) depositados bajo el numero de catalogo CRBMUV 85148.

Con la descripción de estas dos nuevas especies para la ciencia se incrementa a 20 el número de especies válidas, informadas de la región biogeográfica del Chocó colombiano. Hasta la fecha esta área alberga cerca del $55 \%$ de las especies conocidas del género Hypolobocera.

\section{AGRADECIMIENTOS}

El autor agradece a Gilberto Rodríguez (qepd), del Instituto Venezolano de Investigaciones Científicas, su gentil ayuda con la literatura, revisión y comentarios sobre el manuscrito. A Célio Magalhães, del Instituto Nacional de Pesquisas da Amazônia, Brazil, por el envío de literatura. A Rafael Lemaitre, National Museum of Natural History, Smithsonian Institution, Washington, D.C., por depositar parte del material tipo en USNM, ayuda con literatura y su invaluable y constante colaboración. A Michael Alberico (qepd) y Luis 
"Lucho” Neira, por capturar, donar el material e informar acerca de las condiciones ambientales del sitio de recolección. Al personal de técnicos y docentes de la Sección de Biología Marina de la Universidad del Valle, por permitir el trabajo con el material tipo depositado en la Colección de Referencia (CRBMUV). A Juan Valverde (qepd), Luis A. Zapata, Beatriz Beltrán y resto del personal de técnicos y profesionales del Instituto Nacional de Pesca y Acuícultura (INPA), regional de Buenaventura, por su colaboración. Por último a Raúl Ríos quien elaboró las figuras. La revisión y sugerencias por parte de dos revisores anónimos mejoraron la versión final del artículo. Este trabajo es dedicado especialmente a la memoria del maestro y amigo Henry von Prahl (1948-1989) por su invaluable contribución a la sistemática y ecología de los invertebrados marinos y por su gran esfuerzo y dedicación al estudio de los cangrejos de agua dulce de Colombia. Contribución No. 001 de la Fundación Megadiversidad Colombiana, F.M.C. y No. 58 del CIME, Centro de Investigaciones Marinas y Estuarinas de la Universidad del Valle, Cali, Colombia.

\section{RESUMEN}

Se describen dos nuevas especies de cangrejos de agua dulce del género Hypolobocera de la Serranía de Los Paraguas, Cordillera Occidental de Colombia. H. solimani n. sp. está estrechamente relacionada con $H$. alata Campos 1989, pero se puede distinguir de ésta por el margen superior del mero del tercer maxilípedo, el cual es liso; por la forma del ápice del primer gonópodo y la forma del lóbulo interno o papila del canal espermático, la cual es cóncava, levantada y termina bordeada por diminutas papilas o tubérculos redondeados. $H$. triangula n. sp. es muy similar a $H$. rotundilobata Rodríguez 1994, pero se diferencia de ésta por el tamaño y la forma del lóbulo lateral, una cresta basal de tubérculos y la superficie apical del gonópodo. Con estas dos nuevas descripciones se incrementa a 20 el número de miembros válidos del género Hypolobocera conocidos del occidente de Colombia o del Chocó biogeográfico.

Palabras clave: Hypolobocera solimani, Hypolobocera triangula, cangrejo de agua dulce, Colombia, Pseudothelphusidae, especies nuevas.

\section{REFERENCIAS}

Campos, M.R. 1989. Nuevas especies de cangrejos de agua dulce del género Hypolobocera (Crustacea: Decapoda: Pseudothelphusidae) para Colombia. Trianea 3: 143-147.

Campos, M.R. 2003. A review of the freshwater crabs of the genus Hypolobocera Ortmann, 1897 (Crustacea: Decapoda: Brachyura: Pseudothelphusidae), from Colombia. Proc. Biol. Soc. Wash. 116: 754-802.

Campos, M.R. \& G. Rodríguez. 1995. Two new species of freshwater crabs of the genus Hypolobocera from Colombia(Crustacea:Decapoda: Pseudothelphusidae). Proc. Biol. Soc. Wash. 108: 649-655.

Magalhães, C. \& M. Türkay. 1996. Taxonomy of the neotropical freshwater crab family Trichodactylidae. I. The generic system with description of some new genera (Crustacea: Decapoda: Brachyura). Senckenb. Biol. 75: 63-95.

Pretzmann, G. 1965. Verläufiger Berich über die Familie Pseudothelphusidae. Anz. Math. Naturw. Kl. Österr. Akad. Wiss. 1: 1-10.

Pretzmann, G. 1968. Neue südamerikanische Süßwasserkrabben (Pseudothelphusidae). Vourläufige Mitteilung. Entom. Nachrbl. Wien 14: 23-26.

Rathbun, M.J. 1905. Les crabes d'eau douce (Potamonidae). Nouv. Arch. Mus. Hist. Nat. 7: 159-321.

Rodríguez, G. 1980. Description preliminaire de quelques especes et genres nouveaux de crabes d'eau douce de l'Amerique tropicale (Crustacea, Decapoda, Pseudothelphusidae). Bull. Mus. Natl. Hist. Nat. 2, section A(3): 889-894.

Rodríguez, G. 1982. Les crabes d'eau douce d'Amerique, famille des Pseudothelphusidae. Faune Trop. 22: 1-223.

Rodríguez, G. 1992. The freshwater crabs of America. Family Trichodactylidae and supplement to the family Pseudothelphusidae. Faune Trop. 31: 1-189.

Rodríguez, G. 1994. A revision of the type material of some species of Hypolobocera and Ptychophallus (Crustacea, Decapoda, Pseudothelphusidae) in the National Museum of Natural history, Washington D.C., with descriptions of a new species and a new subspecies. Proc. Biol. Soc. Wash. 107: 296-307.

Rodríguez, G. \& R. von Sternberg. 1998. A revision of the freshwater crabs of the family Pseudothelphusidae (Decapoda: Brachyura) from Ecuador. Proc. Biol. Soc. Wash. 111: 110-139. 
Smalley, A. 1964. A terminology for the gonopods of the American river crabs. Syst. Zool. 13: 28-31.

Türkay, M. 1975. Statement: Die Bedeutung des Gonopodenaufbaus für die Aufklärung Verwandtschaftlicher Beziehungen bei dekapoden Crustaceen. Aufs. Red. Senckenb. Naturf. Ges. 27: 114-115.

von Prahl, H. 1983. Hypolobocera gorgonensis sp. nov. (Crustacea: Brachyura: Pseudothelphusidae) un nuevo cangrejo de agua dulce de la Isla Gorgona, Colombia. Cespedesia 12: 105-110.

von Prahl, H. 1985. Distribución del cangrejo de agua dulce Hypolobocera beieri Pretzmann, 1968 y anatomía de su gonópodo. Actual. Biol. 14: 43-47.

von Prahl, H. 1987a. Hypolobocera dentata sp. nov. A new freshwater crab (Crustacea: Brachyura:
Pseudothelphusidae) from the Cordillera Occidental, Colombia. Rev. Biol. Trop. 35: 93-95.

von Prahl, H. 1987b. Redescripción de Hypolobocera buenaventurensis, un cangrejo de agua dulce (Decapoda, Pseudothelphusidae) del Pacífico Colombiano. Bol. Ecotropica 16: 59-64.

von Prahl, H. 1988a. Fresh-water crabs (Crustacea: Decapoda: Pseudothelphusidae) of the Pacific drainage of Colombia. Zool. Jb. Syst. 115: 171-186.

von Prahl, H. 1988b. Cangrejos de agua dulce (Crustacea, Brachyura, Pseudothelphusidae y Trichodactylidae) capturados en el Departamento de Antioquía, Colombia. Bol. Ecotropica 18: 3-17.

von Prahl, H. \& J. Giraldo. 1985. Un nuevo cangrejo de agua dulce de la Cordillera Central de Colombia. Lozania 49: 1-5. 